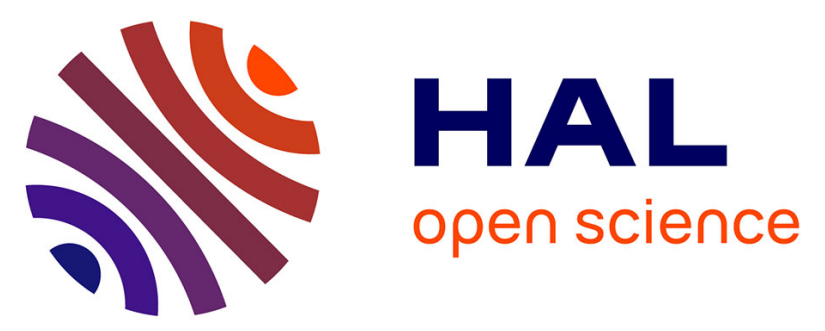

\title{
Charge Transfer Evidence between Carbon Nanotubes and Encapsulated Conjugated Oligomers
}

\author{
Laurent Alvarez, Yann Almadori, Raul Arenal, Moulay-Rachid Babaa, \\ Thierry Michel, Rozenn Le Parc, Jean-Louis Bantignies, Bruno Jousselme, \\ Serge Palacin, Patrick Hermet, et al.
}

\section{To cite this version:}

Laurent Alvarez, Yann Almadori, Raul Arenal, Moulay-Rachid Babaa, Thierry Michel, et al.. Charge Transfer Evidence between Carbon Nanotubes and Encapsulated Conjugated Oligomers. Journal of Physical Chemistry C, 2011, 115, pp.11898-11905. 10.1021/jp1121678 . cea-00960683

\section{HAL Id: cea-00960683 https: / hal-cea.archives-ouvertes.fr/cea-00960683}

Submitted on 13 Dec 2019

HAL is a multi-disciplinary open access archive for the deposit and dissemination of scientific research documents, whether they are published or not. The documents may come from teaching and research institutions in France or abroad, or from public or private research centers.
L'archive ouverte pluridisciplinaire HAL, est destinée au dépôt et à la diffusion de documents scientifiques de niveau recherche, publiés ou non, émanant des établissements d'enseignement et de recherche français ou étrangers, des laboratoires publics ou privés. 


\title{
Charge transfer evidence between carbon nanotubes and encapsulated conjugated oligomers
}

\author{
L. Alvarez ${ }^{(1,2)}$, Y. Almadori ${ }^{(1,2)}$, R. Arenal ${ }^{(3,4)}, \mathrm{R}$. Babaa ${ }^{(5,6)}$, T. Michel $^{(1,2)}, \mathrm{R}$. \\ Le Parc ${ }^{(1,2)}$, J-L. Bantignies ${ }^{(1,2)}$, B. Jousselme ${ }^{(5)}$, S. Palacin ${ }^{(5)}$, P. Hermet ${ }^{(1,2)}$, \\ and J-L. Sauvajol ${ }^{(1,2)}$
}

(1) Université Montpellier 2, Laboratoire Charles Coulomb UMR 5221, F34095, Montpellier, France

(2) CNRS, Laboratoire Charles Coulomb UMR 5221, F-34095, Montpellier, France

(3) Laboratoire d'Etude des Microstructures, CNRS-ONERA, 92322 Chatillon, France

(4) Laboratorio de microscopias avanzadas (LMA), Instituto de Nanociencia de Aragon (INA), U. Zaragoza, c/ Mariano Esquillor s/n, 50018 Zaragoza (Spain)

(5) CEA, IRAMIS, SPCSI, Laboratoire de Chimie des Surfaces et Interfaces, 91191 Gif-sur-Yvette Cedex, France

${ }^{(6)}$ Chemical Engineering Department, University of Technology PETRONAS, (UTP), Ipoh- Perak, Malaysia

\begin{abstract}
Hybrid system consisting in quaterthiophene derivative inserted into carbon nanotubes is studied. Encapsulation efficiency of the conjugated oligomers in the hollow core of nanotube is investigated by Transmission Electron Microscopy and spatial resolved electron energy loss spectroscopy. Infrared spectroscopy evidences a significant positive charge transfer on the inserted oligothiophene. Raman spectra display different behaviours depending on the excitation energy and correlated to the quaterthiophene optical absorption energy. At high excitation wavelength, (far from the oligomer resonance), radial breathing modes exhibit a significant upshift consistent with an encapsulation effect. At low excitation wavelength (close to the oligomer resonance), both the $\mathrm{G}$ band shift and the low frequency modes vanishing suggest a significant charge transfer between the quaterthiophene and the nanotubes.
\end{abstract}

\section{Introduction}


Over the last years, $\pi$ conjugated oligomers and polymers caught considerable attention in the research community as they can play a crucial role for electronic and optoelectronic applications [1-3]. They are easy to manufacture at low costs, lightweight, making them very promising materials for devices for renewable and alternative energy sources [4,5]. They exhibit a great flexibility and are very light allowing various ergonomics [6,7]. Thus, the organic materials display significant potential for competition with silicon in the photovoltaic devices [5]. They however display some weak points like their brittleness and their low electric conductivity [8]. An efficient method to overcome those drawbacks consists in combining species with carbon nanotubes (NT) to create a new hybrid system. Three methods are currently used for that purpose. The first one is a functionalization of polymers at the surface of the tubes [9]. This method has the advantage of producing strong interactions between the two species. On the other hand, it modifies the physical properties of the nanotubes by creating defects on its surface [10]. The second method is the noncovalent adsorption or wrapping of various functional molecules [11]. The third one consists in encapsulating oligomers inside nanotubes in order to keep their original physical properties $[8,12,13]$.

One of the most powerful tools to investigate carbon nanotube optical and electronic properties is Raman spectroscopy. Several spectral ranges in the Raman spectra of carbon nanotubes are of interest [14-15]. They are very sensitive to charge transfers and/or environmental effects, making thus possible to investigate very efficiently the interaction between oligomer and nanotubes.

The first region of interest is located between 100 and $300 \mathrm{~cm}^{-1}$ where radial breathing modes (RBM) related to the tube diameters are observed. The relationship between the frequency $\omega$ and the tube diameter $d$ is usually given by the following equation: $\omega\left(\mathrm{cm}^{-1}\right)=\mathrm{A} / \mathrm{d}(\mathrm{nm})+\mathrm{B}$ where $\mathrm{A}$ and $\mathrm{B}$ are two constants [14-16]. Note that, under doping, a huge decrease or a loss of RBM intensity is observed $[17,18]$.

The second range, around $1600 \mathrm{~cm}^{-1}$, exhibits the tangential modes (G-band). Gmodes display different lineshapes depending on the metallic or semiconducting character of the nanotubes [14-15,19]. Indeed, electron-phonon coupling (EPC) has been evidenced in carbon nanotubes [20-23] and many works have demonstrated that G-modes are very sensitive to EPC [23-26]. Qualitatively, in semiconducting tubes, the profile of each G-band is narrow and symmetric. By contrast, bundles of metallic tubes display a broad low-frequency mode. The measurement of G-band gives therefore some insights on the metallic or semiconducting character of nanotubes under investigations. Furthermore, Raman G band has been shown to undergo a renormalization effect due to EPC [25] so that the phonon energy is reduced. Upon $n$ or $p$ doping, the strength of the EPC decreases leading in both cases to an upshift of the $\mathrm{G}$ band position [25]. One 
important point is that the magnitude of this shift depends on the tube diameter, the larger the diameter the stronger the renormalization effect. On the other hand, a positive (respectively a negative) charging leads to a strengthening (respectively a softening) of the C-C bond and thus to an upshift (respectively a downshift) of the $\mathrm{G}$ band [26]. Thus, for $p$ type doping both effects shift the modes towards higher frequency. By contrast, a negative charging leads to a softening of the mode so that a competition between both effects takes place. Consequently, the direction of the G-band shift allows discriminating between $n$ or $p$ type doping of the nanotubes [26]. In particular, a downshift can only be assigned to a negative charging.

In this work, we study the properties of a hydrid system consisting in a 5,5"'Dimethyl-2,2':5',2":5",2"'-quaterthiophene (4T, figure 1), trapped into carbon nanotubes. This material is called 4T@NT in the following of this article. Transmission Electron Microscopy (TEM) and spatial resolved electron energy loss spectroscopy (SR-EELS) are used to investigate the morphology, the structure, and the chemical composition of the sample. The properties of 4T@NT, and especially the charge transfer between the oligomers and the nanotubes, are investigated by means of Raman and infrared (IR) spectroscopies.

\section{EXPERIMENTAL:}

Sample preparation: Two sources of nanotubes have been used: commercial electric arc single-walled carbon nanotubes, 90\% purified (EA-NT in the following) provided from Nanocarblab [27] and CVD nanotubes (CVD-NT in the following) provided from Cheaptubes Company [28].

Encapsulation of dimethyl-quaterthiophene (4T) into nanotubes (NT) was performed using the vapour reaction method. Such method is similar to the one used for the elaboration of $\mathrm{C}_{60}$-peapods [29]. Before the encapsulation treatment, carbon nanotubes were purified and open via thermal oxidation performed in air at $150^{\circ} \mathrm{C}$ for 30 minutes. Then, NTs were mixed with $4 \mathrm{~T}$ in weight ratio: $\mathrm{w}_{\mathrm{CNT}} / \mathrm{w}_{4 \mathrm{~T}}=0.5$, in a glass tube. The sample was outgased under $4.510^{-6} \mathrm{mbar}$ at ambient temperature for 1 hour followed by a thermal treatment at $100^{\circ} \mathrm{C}$ for 3 hours. Then, NTs with 4T were sealed in glass tube at $4.510^{-6} \mathrm{mbar}$ and heated to $230^{\circ} \mathrm{C}$ for 1 hour. To remove $4 \mathrm{~T}$ adsorbed on the outer walls of NT, the sample was heated at $215^{\circ} \mathrm{C}$ under $4.510^{-6}$ mbar for one hour (sublimation temperature of $4 \mathrm{~T}$ under $4.510^{-6} \mathrm{mbar}$ is $210^{\circ} \mathrm{C}$ ). The sample was then washed with acetone and stored in the oven at $100^{\circ} \mathrm{C}$ for 24 hours.

Raman spectroscopy: Raman experiments were performed on a triple monochromator spectrometer (Jobin Yvon T64000), equipped with a charge- 
coupled detector, in a back scattering geometry, using the $458,488,568,514.5$, 647.1 and $752 \mathrm{~nm}$ excitation wavelengths. In order to avoid the heating of the tubes and oligomers, the laser power was adjusted at $700 \mu \mathrm{W}$ with a spot diameter of about $3 \mu \mathrm{m}$ using a $50 \mathrm{X}$ objective. The resolution is about $2 \mathrm{~cm}^{-1}$.

Raman experiments with the $1064 \mathrm{~nm}$ excitation wavelength and photoluminescence spectra were measured using a Bruker RFS100 FT Raman spectrometer equipped with a nitrogen-cooled Germanium detector. The excitation source is a continuous Nd-YAG laser emitting at $1.16 \mathrm{eV}(1064 \mathrm{~nm})$. Excitation at $1.16 \mathrm{eV}$ allows the observation on the same spectra of the PL signal and the Stokes Raman signal (Raman shift in the range 100 and $3200 \mathrm{~cm}^{-1}$. The resolution is about $4 \mathrm{~cm}^{-1}$. For photoluminescence spectra, nanotubes have been dispersed in an aqueous solution with SDBS to obtain individual nanotubes.

Infrared spectroscopy: Fourier transform infrared (FTIR) experiments were carried out on a Bruker IFS $66 \mathrm{~V}$ spectrometer equipped with a $\mathrm{N}_{2}$-cooled MCT (Mercury Cadmium Telluride) detector. Transmission IR spectra were recorded in the $400-4000 \mathrm{~cm}^{-1}$ range. The spectral resolution was $2 \mathrm{~cm}^{-1}$ and 64 scans were co-added for each spectrum.

TEM and SR-EELS: The TEM samples were prepared by dispersing the NTs powders in ethanol. The dispersions were ultrasonicated and subsequently deposited on a holey carbon $3 \mathrm{~mm}$ copper grids.

High resolution TEM was performed using a FEI Tecnai F20 and FEI CM20 microscopes operating both of them at $200 \mathrm{keV}$.

Spatial resolved EEL spectra were recorded using a VG-HB501 dedicated scanning transmission electron microscope (STEM) instrument equipped with a cold field emission gun (FEG), operated at $100 \mathrm{keV}$ with an energy resolution close to $0.7-0.8 \mathrm{eV}$ in the core-loss region. Convergence angle on the sample and collection angle of the spectrometer were 15 and $24 \mathrm{mrad}$, respectively. This spectroscopic information was obtained using the spectrum-imaging (SPIM) acquisition mode [30-33].

\section{Results and discussion:}

TEM and SR-EELS studies were performed on 4T@CVD-NT. This sample contains both multi-walled (MWNT) and single walled (SWNT) nanotubes. Figure 2a corresponds to an HR-TEM micrograph of 4T@NT sample. This micrograph clearly displays a double-walled nanotube filled with presumably 4T. This image is a very significant point. In fact, in the literature there are several examples showing similar HRTEM micrographs of filled NTs. However, 
as it is well-known in the field of electron microscopy in NTs, this conclusion might be an over-interpretation of the data because from this kind of HRTEM micrographs it is not possible to deduce the structure of this material, neither to obtain any chemical information. Thus, in order to go further in the analysis of these materials and to know where the 4T molecules are localized we have developed SR-EELS. This technique is a very powerful tool to investigate such nanomaterials at (sub-)nanometer scale [31].

First investigations concern individual NT (Figure 2b, bright field (BF) image), acquiring a spectrum-image (SPIM) in the whole area showed in the high angle annular dark field (HAADF) image (Figure 2c). Figure $2 \mathrm{~d}$ shows the intensity profiles of $\mathrm{C}$ and $\mathrm{S}$ extracted from the two chemical maps (C and $\mathrm{S}$ ) obtained in the marked region of Figure 2c. From these intensity profiles, we can conclude that sulphur atoms are present inside the NT. Thus, these profiles clearly demonstrate that sulphur atoms from the conjugated oligomers (4T) are mainly localized within few nanometers inside the NT, at its apex.

Structural and chemical properties of bundle of single-walled nanotubes have also been investigated (Figure 3). Figure 3a corresponds to an HAADF image acquired in parallel with the SPIM. Chemical maps of carbon and sulphur (Figures $3 b$ and $3 c$ ) have been extracted from a 20x20 SPIM. Figure 3d shows two EEL spectra. Each of these two EEL spectra corresponds to the sum of 9 spectra ( $3 \times 3$ probe positions of the SPIM) recorded in the areas marked on Figure 3a. The energy loss near-edge feature (ELNES) of the C-K edge consisted of a $\pi^{*}$ peak at $\sim 285 \mathrm{eV}$ and a well-defined $\sigma^{*}$ band starting at $\sim 292$ $\mathrm{eV}$ [30-33]. The $\mathrm{S}-\mathrm{L}_{2,3}$ edge consist in a rather large peak around $165-220 \mathrm{eV}$. Carbon $\mathrm{K}$ edge at $\sim 284 \mathrm{eV}$ is present in both of these two EEL spectra. These fingerprints are typical for the $\mathrm{sp}^{2}$ hybridization of the $\mathrm{C}$ atoms in a graphitic network, and indicate that the SWNTs are well crystallized. $\mathrm{L}_{2,3}$ edge of sulphur is clearly visible in Figure 3d(i). However, in the second EEL spectra of Figure $3 \mathrm{~d}$ (ii), the quantity of S (if any) is very low. This means that sulphur (from 4T) is not surrounding the bundles but mainly localized within the bundles, either inside the SWNT or in the interstitial sites in between three tubes. The sulphur chemical map confirms these results (Figure 3c). However, considering the 4T diameter $(0.48 \mathrm{~nm})$, the insertion into the free space $(\mathrm{d}=0.40 \mathrm{~nm}$ for a tube diameter of $2 \mathrm{~nm}$ ) in between three tubes is not likely and even impossible for smaller diameter tubes (for instance, the free space for a $1.5 \mathrm{~nm}$ tube diameter is $0.31 \mathrm{~nm}$ ). We thus assume that $4 \mathrm{~T}$ is localized in the hollow core of SWNTs. It is worth noting that the $\mathrm{S}$ distribution/repartition is rather uniform for the whole analyzed area of the bundle.

Figure 4 displays the photoluminescence spectra of aqueous solution of individual pristine CVD-NT and 4T@CVD-NT samples wrapped in SDBS recorded with an excitation wavelength of $1064 \mathrm{~nm}$. The peak assignments of 
the different nanotubes have been derived from the work of S. Bachilo et al [35]. A clear redshift is observed between both samples. Such a redshift has been previously interpreted as an encapsulation effect of different molecules such as metallocenes [36] or squarylium dye [37], inside carbon nanotubes. It is noteworthy that considering the $4 \mathrm{~T}$ and the nanotube diameters (respectively 0.48 and $0.84 \mathrm{~nm}$ for the smallest $(8,4)$ nanotube) and taking into account the van der Waals radius of a carbon nanotube wall $(\sim 0.16 \mathrm{~nm})$, all the nanotubes are expected to be filled and undergone the redshift as observed on figure 4 . This result is another insight on the efficiency of the encapsulation process.

Figure 5a displays the IR spectra of EA-NT, 4T and 4T@EA-NT. The IR active modes of NT are discussed elsewhere [38, 39]. The expected IR absorption bands of the dimehyl-quaterthiophene are the following [40,41]. The bands at 1513 and $1445 \mathrm{~cm}^{-1}$ are associated respectively to the anti-symmetric and the symmetric $\mathrm{C}=\mathrm{C}$ stretching modes. The absorption band at $1279 \mathrm{~cm}^{-1}$ corresponds to anti-symmetric in-plane $\mathrm{C}-\mathrm{H}$ bending. The doublet at 1217 and $1166 \mathrm{~cm}^{-1}$ arises from the inter ring CC stretching vibration. The band at 1198 $\mathrm{cm}^{-1}$ can be probably related to anti-symmetric stretching of the two-end $\mathrm{C}_{\alpha}-\mathrm{CH}_{3}$ bonds. Peaks at 1066 and $1042 \mathrm{~cm}^{-1}$ correspond to in-plane $\mathrm{C}-\mathrm{H}$ bending. The multiplet of bands at 899 and $843 \mathrm{~cm}^{-1}$ arise from the symmetric and antisymmetric C-S stretching modes. The absorption bands at 795 and $787 \mathrm{~cm}^{-1}$ are assigned to the out-of-plane $\mathrm{C}-\mathrm{H}$ bending vibrations. The weak band at $644 \mathrm{~cm}^{-1}$ is associated to an in-plane ring deformation mode.

The oligomer IR active modes and those of nanotubes are observed in the IR spectrum of the 4T@NT sample (fig5a). However, insertion into nanotube clearly leads to significant modifications of the infrared modes of the oligomer, especially in the $600-900 \mathrm{~cm}^{-1}$ and $1400-1600 \mathrm{~cm}^{-1}$ spectral ranges. Upon encapsulation, the intensity of some $4 \mathrm{~T}$ modes significantly decreases and the frequency of the most intense band round $800 \mathrm{~cm}^{-1}$ is significantly downshifted and broadened. Figure $5 \mathrm{~b}$ compares the IR spectra of $4 \mathrm{~T}$ (circles, topmost) and 4T@EA-NT (triangles, bottommost) together with the IR spectra of raw and iodine doped 4T (grey lines) [40]. One can first check that the spectra corresponding to the pristine 4T measured in this work (circles) and in ref [40] (curve a) are quite similar. The iodine doping of 4T leads to the progressive vanishing of the main modes as a function of the doping level. The downwards arrows indicate the peaks that are disappearing upon doping. Figure $5 \mathrm{c}$ details the IR spectra in the $950-600 \mathrm{~cm}^{-1}$ range of the pristine $4 \mathrm{~T}$ and $4 \mathrm{~T}$ inserted into EA-NT. The most intense band in $4 \mathrm{~T}$ contains three modes located at 786, 795 and $800 \mathrm{~cm}^{-1}$. In 4T@EA-NT sample, only a broad and downshifted single band is measured at $786 \mathrm{~cm}^{-1}$. It must be pointed out that the same behavior is found in iodine doped $4 \mathrm{~T}$ (see figure $5 \mathrm{~b}$ (curve $\mathrm{d}$ ) and the table 1 of reference [40]). 
For instance, a peak around $670 \mathrm{~cm}^{-1}$ which appears on iodine doped $4 \mathrm{~T}$ is observable on figure $5 \mathrm{c}$.

In summary, we found out a lot of similarities between the IR spectra of 4T inserted into NT and iodine doped 4T. Iodine is well known to be an electron acceptor leading to a positive charging 4T in iodine doped 4T sample. The similarities between the IR spectrum of 4T@NT and I@4T sample state that 4T oligomers undergo a significant positive charge transfer when they are trapped inside nanotubes.

To confirm this assumption, we performed Raman spectroscopy to investigate carbon nanotubes behaviour after 4T encapsulation. The Raman spectrum of the $4 \mathrm{~T}$ excited at $647.1 \mathrm{~nm}$ gives rise to several modes in the $600-1600 \mathrm{~cm}^{-1}$ spectral range (Figure 6, bottom). The main features are the following: weak lines at 1564,1533 and $1503 \mathrm{~cm}^{-1}$ probably related to $\mathrm{C}=\mathrm{C}$ vibrations localized at the two outer thiophene rings; 1482 (its shoulder at 1469) and $1448 \mathrm{~cm}^{-1}$ peaks assigned to $\mathrm{C}=\mathrm{C}$ stretching modes; the 1261 and $1224 \mathrm{~cm}^{-1}$ lines associated to inter-ring $\mathrm{C}-\mathrm{C}$ stretching modes; the $1165 \mathrm{~cm}^{-1}$ contribution is assigned to outerring $\mathrm{C}-\mathrm{C}$ bond; the 1058 and $1044 \mathrm{~cm}^{-1}$ lines corresponding to in plane $\mathrm{C}-\mathrm{H}$ deformation; the 736 and $694 \mathrm{~cm}^{-1}$ peaks related to $\mathrm{C}-\mathrm{S}$ stretching modes [40,41]. It must be emphasized that a huge photoluminescence signal prevents the study of the 4T vibrations in the Raman spectrum excited at $514.5 \mathrm{~nm}(2.41$ $\mathrm{eV})$.

The Raman spectrum of 4T@NT excited at $647.1 \mathrm{~nm}$ and $514.5 \mathrm{~nm}$ are displayed on figure 6 . Vibrations of the trapped oligomers are observed in both spectra. It must be pointed out that the huge photoluminescence which prevents the measurement of the 4T Raman spectrum at $514.5 \mathrm{~nm}$ is bleached when 4T molecules are inserted into nanotubes (fig.6, top) making the oligomer Raman features observable at this excitation wavelength with a relative strong intensity (fig.6, top spectrum). The photoluminescence bleaching is another hint of a significant interaction between $4 \mathrm{~T}$ and the nanotubes. Furthermore, this quenching also suggests that the amount of free oligomer molecules within our samples is completely negligible.

Figure 7a displays the Raman spectra of raw (bottom curves) and 4T@NT (top curves) for the CVD-NT samples at two different excitation wavelengths (488 and $514.5 \mathrm{~nm}$ ). The $4 \mathrm{~T}$ encapsulation clearly gives rise to an important loss of the RBM intensities and to a slight downshift of the $\mathrm{G}$ band. If both behaviours are consistent with a charge transfer $[17,18,25,26]$, the $\mathrm{G}$ band downshift can only be explained by a negative charge transfer on the nanotubes [26], in good agreement with IR results which suggest a positive charge transfer on the 4T. However, as reported in the TEM section, CVD-NT sample contains both single and multi-walled nanotubes, exhibits a quite large diameter distribution (from 1 
to $2 \mathrm{~nm}$ ), and thus contain both semiconducting and metallic nanotubes. Such a mixture could hinder our interpretation. Thus, the Raman studies have been focused on the EA-NT samples in the following.

Figure 7b displays the Raman spectra of raw (bottom curves) and 4T@NT (top curves) for the EA-NT samples at different excitation wavelengths. This sample contains no or negligible amount of MWNT and exhibits a rather narrow diameter distribution $(1.44 \pm 0.22 \mathrm{~nm})$ according to the analysis of the RBM measured below $300 \mathrm{~cm}^{-1}$. Thus, selecting the excitation wavelength properly allow probing either semiconducting or metallic nanotubes. It turns out that at least two distinct Raman signatures are displayed depending on the excitation wavelength.

Indeed, the Raman responses of 4T@NT for low excitation wavelengths (514.5, 488 and $458 \mathrm{~nm}$ ) are very similar to those obtained with the CVD-NT since the $4 \mathrm{~T}$ encapsulation leads to the vanishing of the RBM and to a $\mathrm{G}$ band shift (from 1 to $3 \mathrm{~cm}^{-1}$ ). In fact, the direction of the shift (up) is unlike the CVD-NT sample (down). This difference can be easily explained by considering the difference in tube diameters. Large diameters give rise to stronger EPC, so that the renormalization effect is more important than the softening of the modes under negative charge transfer [26], unlike the small diameter tubes, in good agreement with the Raman data obtained respectively on the EA-NT and the CVD-NT.

For 1064 and $752 \mathrm{~nm}$, the $4 \mathrm{~T}$ encapsulation mainly gives rise to the following changes i) tiny or no modification of the $G$ band profile ii) slight modifications of the RBM profile iii) significant upshift of the RBM (from 3 to $10 \mathrm{~cm}^{-1}$ ). This latest point is consistent with a filling effect of the nanotubes, leading to a strengthening of the interatomic force constants [8]. The first two points rather indicate a very weak charge transfer between $4 \mathrm{~T}$ and nanotubes since RBM are expected to vanish and the $G$ band should shift under charge transfer $[17,18]$ [25]. Those results are in good agreement with previous studies on sexithiophene encapsulated into nanotubes $[8,13]$. However, we mentioned from IR experiments a significant positive charge transfer on the conjugated polymers. In fact, considering here an average tube diameter of $1.35 \mathrm{~nm}$ deduced from the RBM frequency, the number of carbon atoms surrounding a $4 \mathrm{~T}$ molecule (20 nm length) is about 400 . We can thus infer that the charge transfer remains weak for the nanotubes, leading to tiny modifications of the EA-NT Raman spectra.

The Raman spectrum recorded with the $647.1 \mathrm{~nm}$ excitation wavelength is quite peculiar. Indeed, most of the modifications are similar to those observed at 1064 and $752 \mathrm{~nm}$, but the very important shift $\left(10 \mathrm{~cm}^{-1}\right)$ of the RBM is also observed on the $\mathrm{G}$ band. Furthermore, the 2D band undergoes a shift of about $20 \mathrm{~cm}^{-1}$ (not shown), meaning that the modes undergo a rigid upshift, unlike what is observed with the others excitation wavelength. We have no hypothesis to account for this 
behaviour. Anyway, we assume at $647.1 \mathrm{~nm}$ a weak charge transfer, as observed at 1064 and $752 \mathrm{~nm}$.

To summarize, two Raman regimes are displayed as a function of the excitation wavelength. The first one, from 514.5 to $458 \mathrm{~nm}$ gives rise to important modifications that suggest a significant charge transfer between both systems. The second one, from 647.1 to $1064 \mathrm{~nm}$, is consistent with a filling of the nanotubes and a weak charge transfer between $4 \mathrm{~T}$ and NTs. By the way, the spectrum recorded at $568 \mathrm{~nm}$ displays an intermediate behaviour, with no shift of the $\mathrm{G}$ band, a significant shift of the RBM and an important loss of the RBM intensity. Furthermore, decreasing the excitation wavelength obviously leads to an important enhancement of the 4T Raman signal (in the $1400-1500 \mathrm{~cm}^{-1}$ range) with respect to the nanotubes $\mathrm{G}$ band. The $4 \mathrm{~T}$ Raman modes intensity certainly increases because the excitation energy is getting closer to the $4 \mathrm{~T}$ optical absorption energy (close to $400 \mathrm{~nm}$ or $3 \mathrm{eV}$ ). We thus claim that increasing the excitation energy gives rise to a photo-induced charge transfer between nanotubes and the oligomer. Consequently, the two Raman regimes could actually correspond to in and out of resonance of the $4 \mathrm{~T}$ molecules.

\section{Conclusion}

Confinement of dimethyl-quaterthiophene into carbon nanotubes was investigated using HR-TEM, SR-EELS, IR and Raman spectroscopies. From SR-EELS we detected sulphur inside NTs. Both Raman and IR spectroscopy have provided valuable information about the interaction between the 4T molecules and nanotubes. IR spectra of the 4T@NT exhibits many similarities with those of iodine doped $4 \mathrm{~T}$, suggesting a significant positive charge transfer of the oligomers trapped into nanotubes. Furthermore, a detailed analysis of the Raman $\mathrm{G}$ band before and after the oligomer insertion inside nanotubes states a negative charge transfer of the nanotubes. However, Raman spectra suggest that nanotubes, independently on their metallic or semiconducting character, are only slightly affected by this charge transfer when the excitation energy of the laser is far away from the 4T optical absorption energy. By contrast, a significant charge transfer has been evidenced when the excitation energy is getting close to this absorption energy and assigned to a photo induced charge transfer. Experiments are in progress to light the photo induced process.

\section{ACKNOWLEDGMENTS}

The EELS measurements have been developed at the STEM group of the Laboratoire de Physique des Solides (LPS) at the University of Paris-Sud (Orsay 
- France). We acknowledge this group for their support and especially Odile Stephan for fruitful discussions.

The authors would like to thank Prof. Juan T. López Navarrete for his kind authorization of utilizing his data on iodine doped dimethyl-quaterthiophene. 
Figures:

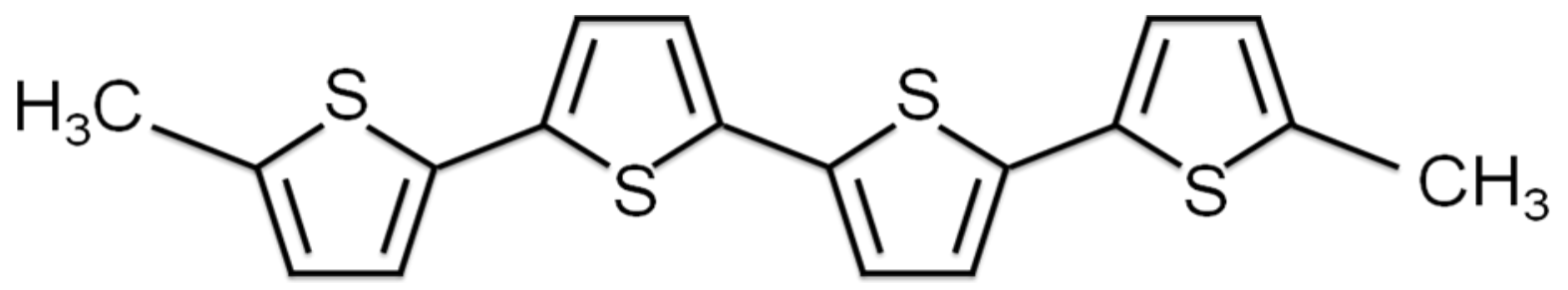

Figure 1: Structure of the 5,5"'-Dimethyl-2,2':5',2":5",2"'-quaterthiophene called for simplicity dimethyl-quaterthiophene (4T) in the text.

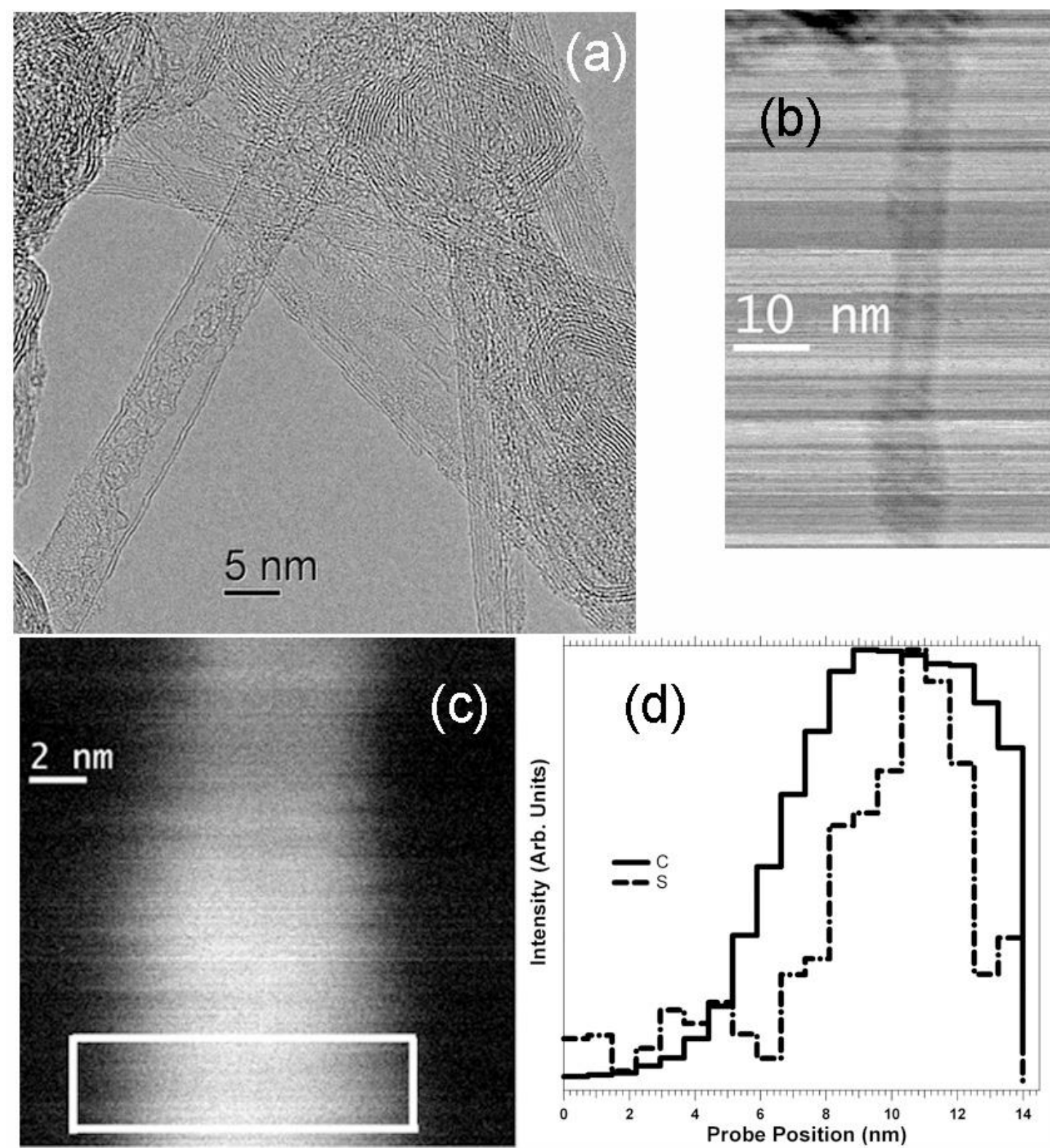


Figure 2: (a) HRTEM micrograph of a filled double-walled nanotube. (b) BF image of a MWNT. (c) HAADF image of this MWNT where a 24x24 SPIM has been recorded. (d) Intensity profile of $\mathrm{C}$ and $\mathrm{S}$ in the region marked in Figure $2 \mathrm{~b}$. These intensity profiles have been extracted from the $\mathrm{C}$ and $\mathrm{S}$ elemental maps that have been obtained from the analysis of the SPIM.
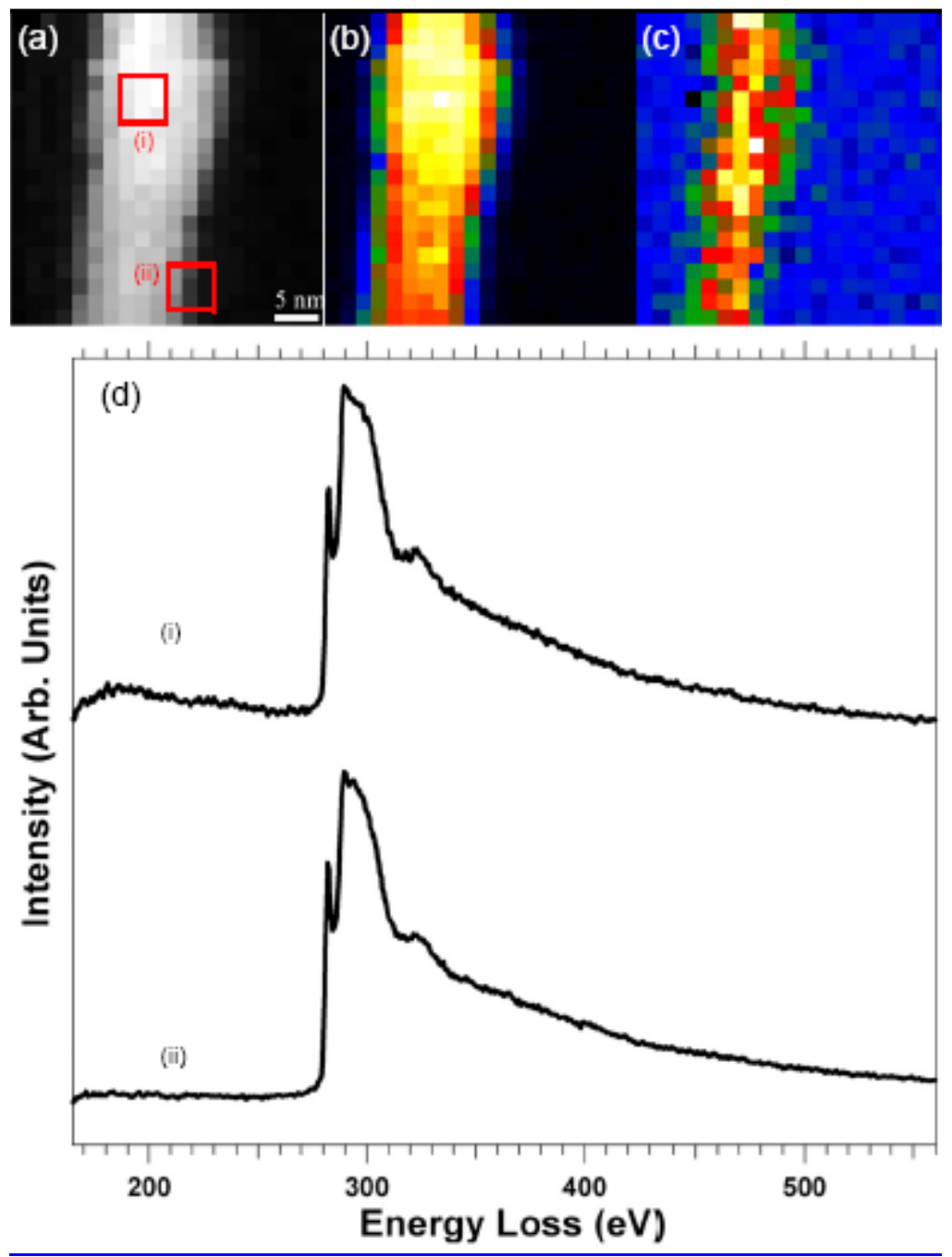

Figure 3: (a) HAADF image of a bundle of SWNTs on which we collected a 20x20 SPIM. (b) carbon and (c) sulphur elemental maps, respectively, extracted from the SPIM. (d) Two spectra from the sum of $9(3 \times 3)$ EEL spectra extracted from the marked areas of Figure $3 \mathrm{a} . \mathrm{K}$ edge of carbon ( $284 \mathrm{eV})\left((\mathrm{i})\right.$ and (ii)) and $\mathrm{L}_{2,3}$ edge of sulphur ( 165-220 eV) only in (i). 


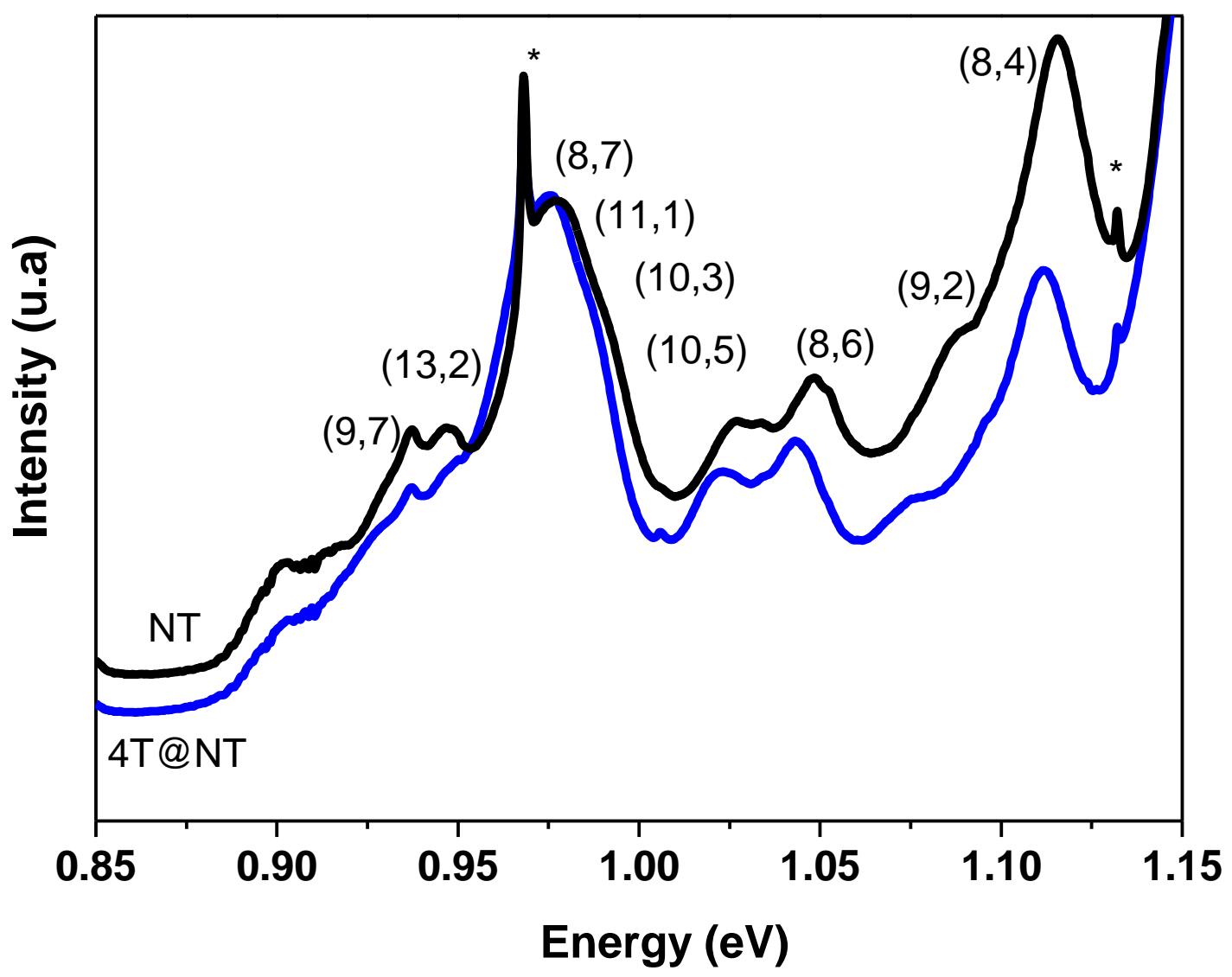

Figure 4: The superimposed Raman and Photoluminescence spectra recorded at $1064 \mathrm{~nm}$ excitation wavelength of aqueous suspensions with SDBS of pristine NT (blue open circles) and 4T@NT(CVD) (black line) samples. Stars indicate the Raman modes of nanotubes. 


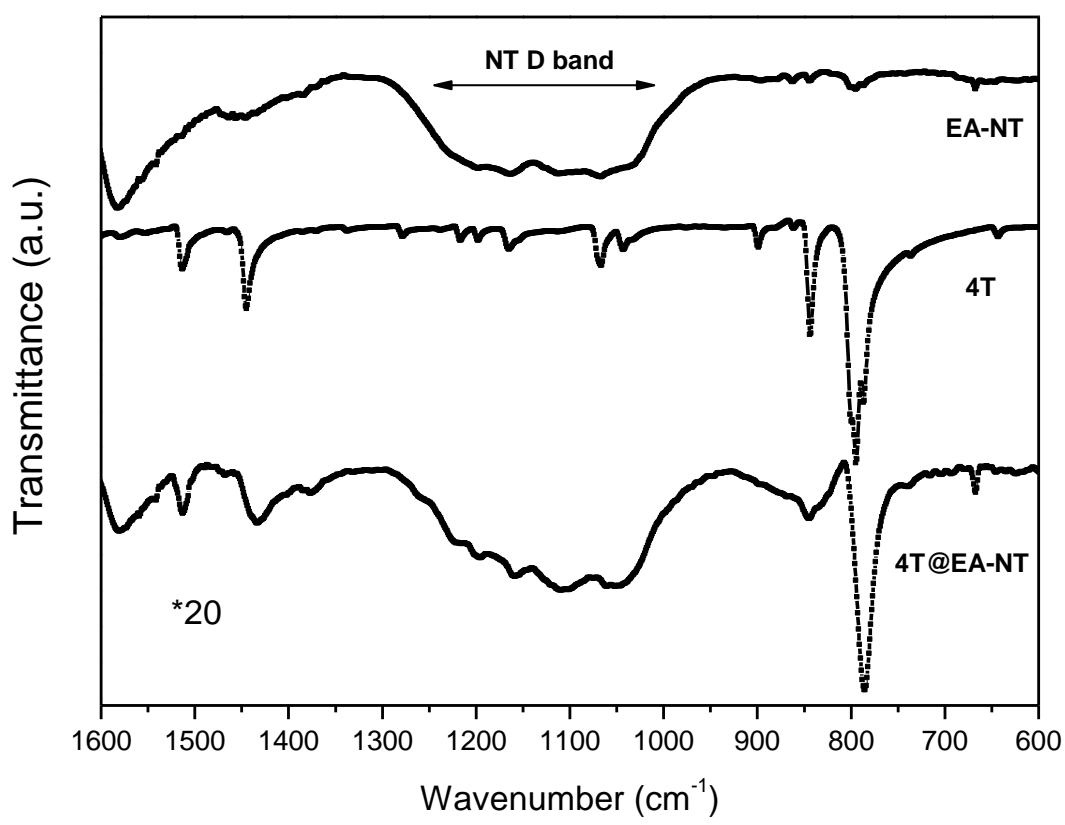

Figure 5a: Infrared spectra of pristine EA-NT (top curve), pristine 4T (middle curve) and 4T @NT (bottom curve). The spectra are vertically translated for clarity and the intensity of the $4 \mathrm{~T} @ \mathrm{NT}$ spectrum is multiplied by 20. 


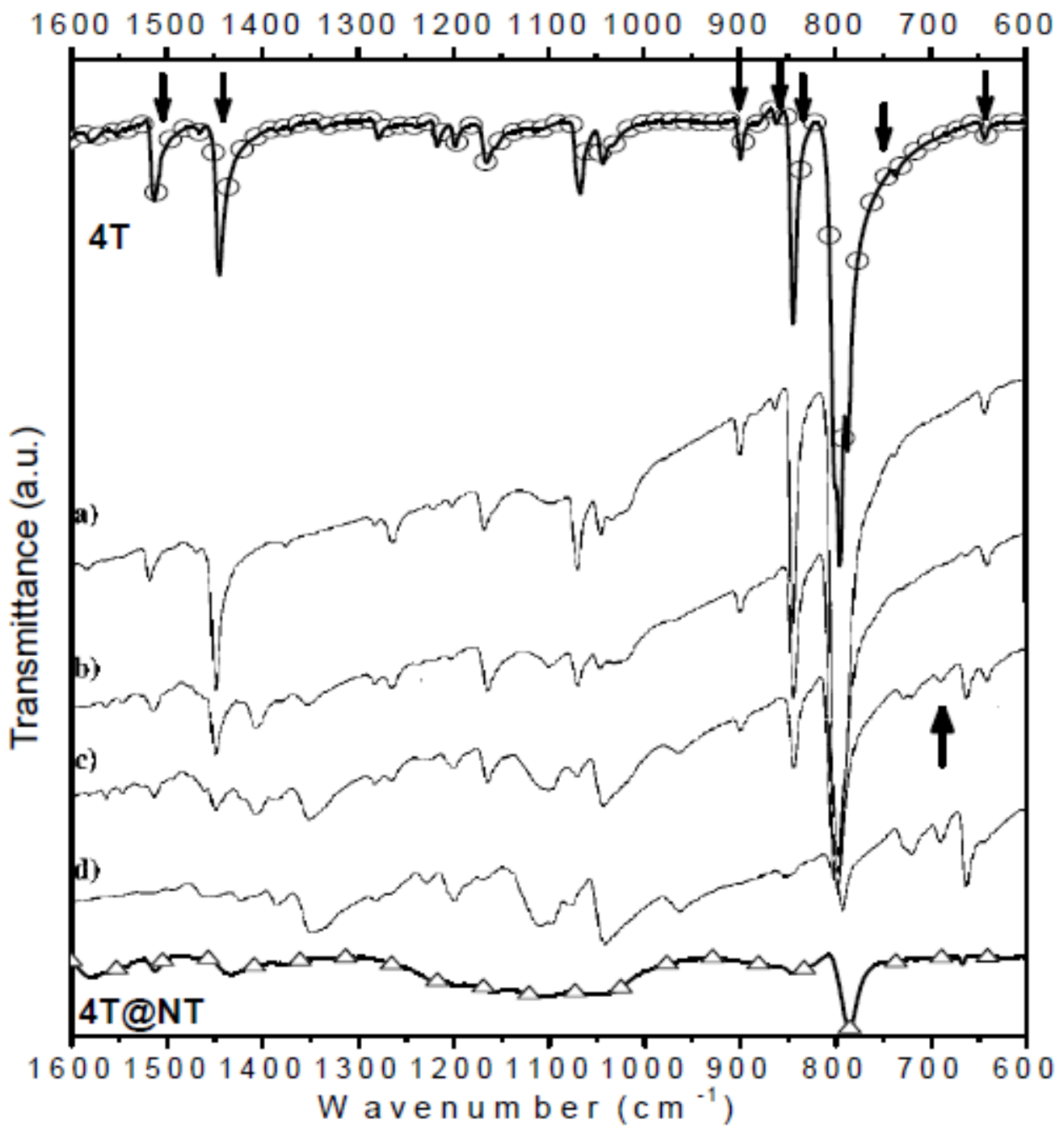

Figure 5b: Infrared Transmittance spectra of raw 4T (open circles, top) and 4T inserted NTs (triangles, bottom) together with iodine doped 4T (grey lines) from [39] ( spectra a, b, c, d correspond respectively to pristine (a) and time doping (td) of 7, 30 minutes and $\mathrm{td}>1$ hour), with the kind authorization of Prof. Juan T. López Navarrete. The spectra are vertically translated for clarity. Arrows indicate the IR peaks of 4T mainly affected by the encapsulation process. 


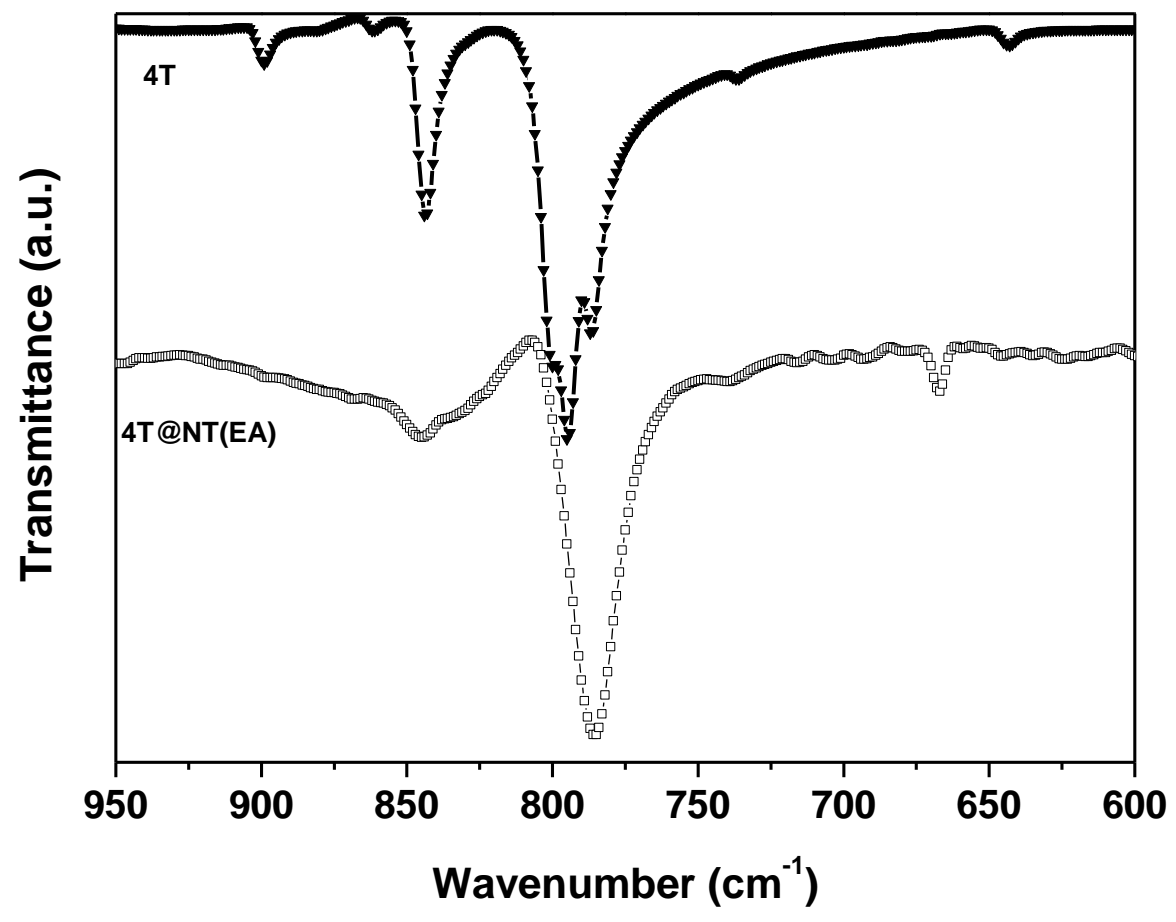

Figure 5c: Infrared spectra of raw 4T (full triangles) and 4T@NT (open squares). The spectra are vertically translated for clarity. 


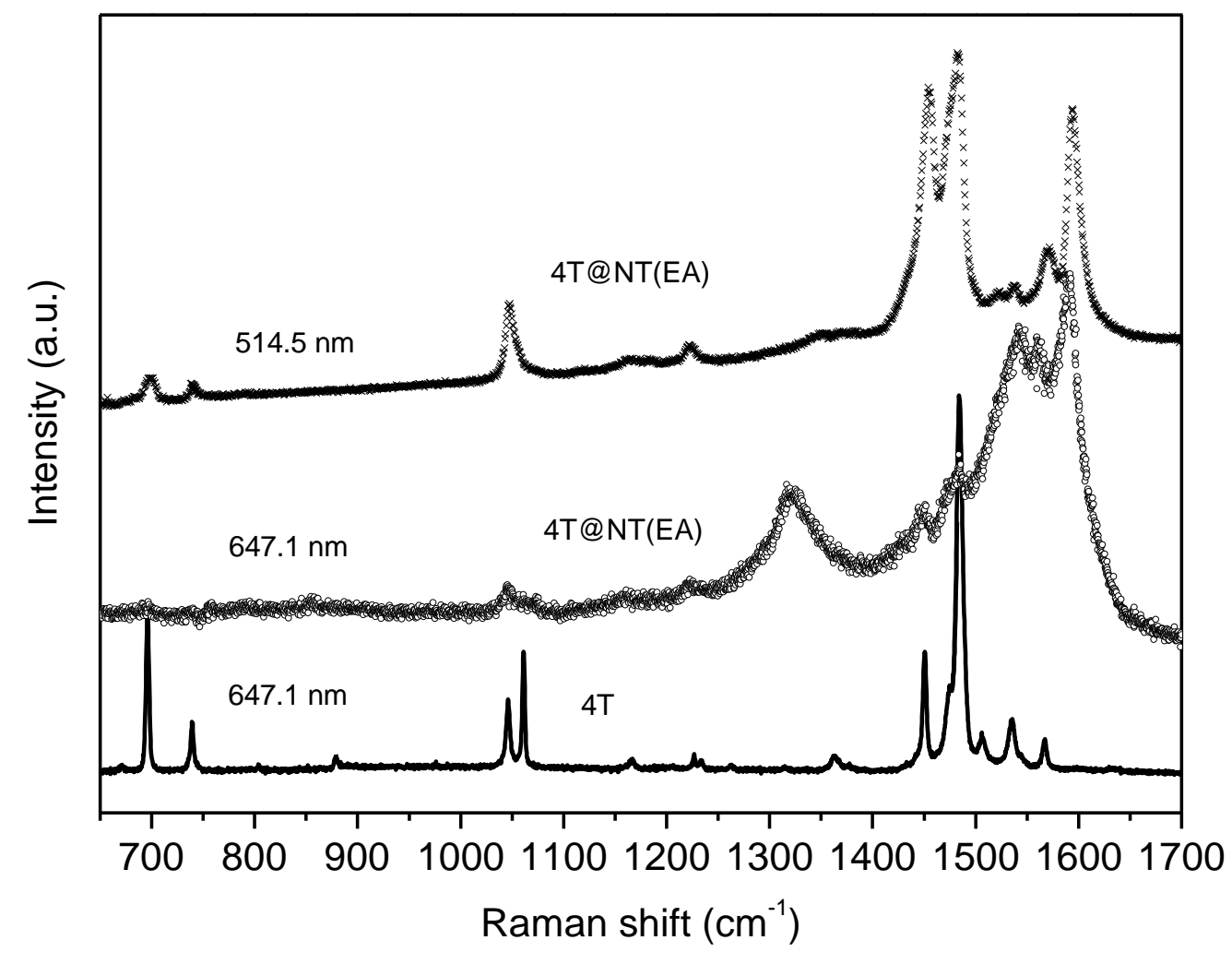

Figure 6: Raman spectra of pristine 4T recorded at an excitation wavelength of $647.1 \mathrm{~nm}$ (bottom) and of 4T@NT(EA) recorded excitation wavelengths of $647.1 \mathrm{~nm}$ (middle) and $514.5 \mathrm{~nm}$ (top) respectively. 


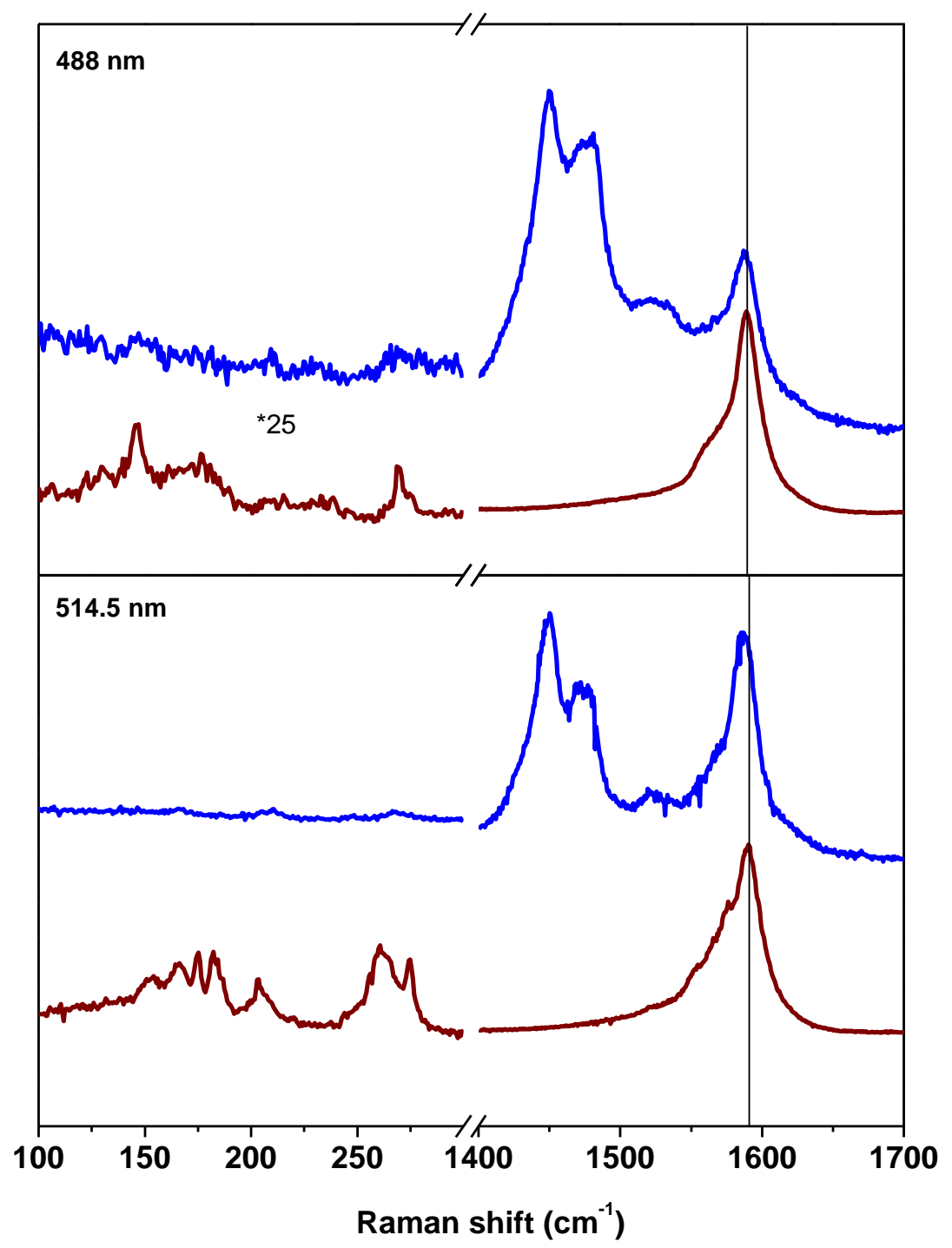

Figure 7a: Raman spectra of raw NT (bottom, wine curve) and 4T inserted NTs (top, blue curve) for the CVD-NT sample for two different excitation wavelengths (514.5 and $488 \mathrm{~nm}$ ). Spectra are normalized on the nanotubes $G$ band intensity. With $488 \mathrm{~nm}$ as excitation wavelength, RBM intensity of both pristine CVD-NT and 4T@EA-NT has been multiplied by 25 for clarity. 


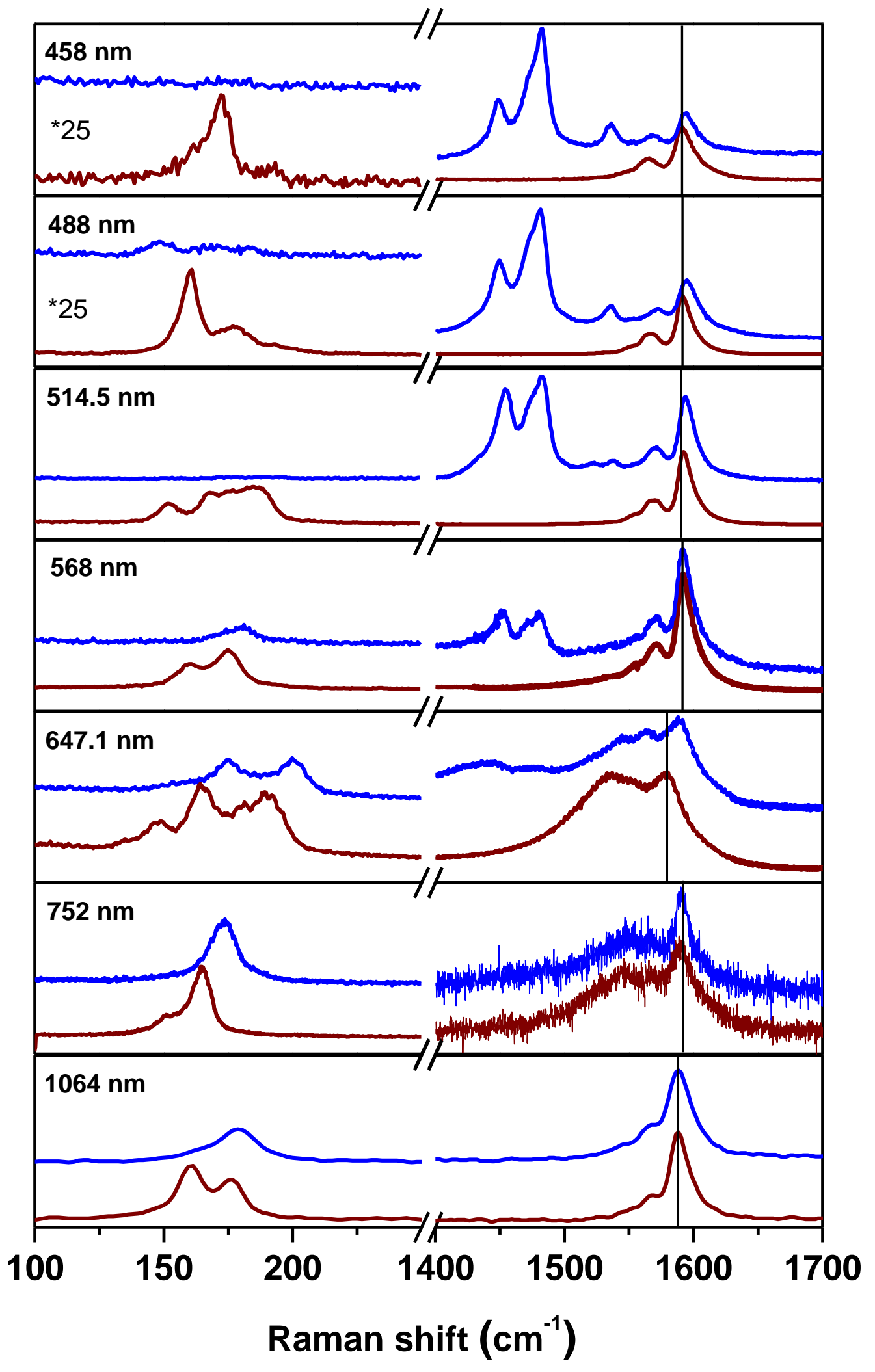


Figure 7b: Raman spectra of raw NT (bottom, wine curve) and 4T inserted NTs (top, blue curve) for the EA-NT for different excitation wavelengths. Spectra are normalized on the nanotubes $G$ band intensity. The spectrum of the $G$ band at $752 \mathrm{~nm}$ is noisy because of the CCD cut-off. With 458 and $488 \mathrm{~nm}$ as excitation wavelengths, RBM intensity of both EA-NT and 4T@EA-NT has been multiplied by 25 for clarity. 


\section{References:}

(1) Berson, S.; Cecioni, S.; Billon, M.; Kervella, Y.; de Bettignies, R.; Bailly, S.; Guillerez, S. Sol. Energy Mater. Sol. Cells 2010, 94, 699-708.

(2) Videlot-Ackermann, C.; Zhang, J.; Ackermann, J.; Brisset, H.; Didane, Y.; Raynal, P.; El Kassmi, A.; Fages, F. Curr. Appl. Phys. 2009, 9, 26-33.

(3) Clarke, T. M.; Gordon, K. C.; Chan, W. S.; Phillips, D. L.; Wagner, P.; Officer, D. L. ChemPhysChem 2006, 7, 1276-1285.

(4) Park, J. H.; Kim, J. S.; Lee, J. H.; Lee, W. H.; Cho, K. J. Phys. Chem. C 2009, 113, 1757917584.

(5) Stylianakis, M. M.; Mikroyannidis, J. A.; Kymakis, E. Sol. Energy Mater. Sol. Cells 2010, 94, 267-274.

(6) El Amrani, A. Ph.D Thesis 2008, University of Limoges.

(7) Lucas, B.; El Amrani, A.; Chakaroun, M.; Ratier, B.; Antony, R.; Moliton, A. Thin Solid Films 2009, 517, 6280-6282.

(8) Loi, M. A.; Gao, J.; Cordella, F.; Blondeau, P.; Menna, E.; Bartova, B.; Hebert, C.; Lazar, S.; Botton, G. A.; Milko, M.; Ambrosch-Draxl, C. Adv. Mater. 2010, 22, 1635-1639.

(9) Babaa, M. R.; Bantignies, J. L.; Alvarez, L.; Parent, P.; Le Normand, F.; Gulas, M.; Mane, J. M.; Poncharal, P.; Doyle, B. P. J. Nanosci. Nanotechnol. 2007, 7, 3463-3467.

(10) Song, M.; Wang, X. M.; Liu, W.; Zuo, J. L. J. Colloid Interface Sci. 2010, 343, 48-51.

(11) Roquelet, C.; Lauret, J. S.; Alain-Rizzo, V.; Voisin, C.; Fleurier, R.; Delarue, M.; Garrot, D.; Loiseau, A.; Roussignol, P.; Delaire, J. A.; Deleporte, E. ChemPhysChem 2010, 11, 1667-1672.

(12) Malard, L. M.; Nishide, D.; Dias, L. G.; Capaz, R. B.; Gomes, A. P.; Jorio, A.; Achete, C. A.; Saito, R.; Achiba, Y.; Shinohara, H.; Pimenta, M. A. Phys. Rev. B 2007, 76, 233412.

(13) Kalbac, M.; Kavan, L.; Gorantla, S.; Gemming, T.; Dunsch, L. Chem. Eur. J. 2010, 16, $11753-$ 11759.

(14) Dresselhaus, M. S.; Dresselhaus, G.; Saito, R.; Jorio, A. Physics Reports 2005, 409, 47-99.

(15) Thomsen, C.; Reich, S.; Maultzsch, J. Philos. Trans. R. Soc. A 2004, 362, 2337-2359.

(16) Araujo, P. T.; Pesce, P. B. C.; Dresselhaus, M. S.; Sato, K.; Saito, R.; Jorio, A. Physica E 2010,

$42,1251-1261$.

(17) Harley, E. C. T.; McNeil, L. E. J. Phys. Chem. Solids 2004, 65, 1711-1718.

(18) Chen, G.; Furtado, C. A.; Kim, U. J.; Eklund, P. C. Phys. Rev. B 2005, 72, 155406.

(19) Jorio, A.; Saito, R.; Dresselhaus, G.; Dresselhaus, M. S. Philos. Trans. R. Soc. A 2004, 362, $2311-2336$

(20) Kataura, H.; Kumazawa, Y.; Maniwa, Y.; Umezu, I.; Suzuki, S.; Ohtsuka, Y.; Achiba, Y. Synth. Met. 1999, 103, 2555-2558.

(21) Jorio, A.; Souza, A. G.; Dresselhaus, G.; Dresselhaus, M. S.; Swan, A. K.; Unlu, M. S.;

Goldberg, B. B.; Pimenta, M. A.; Hafner, J. H.; Lieber, C. M.; Saito, R. Phys. Rev. B 2002, 65, 155412.

(22) Piscanec, S.; Lazzeri, M.; Mauri, F.; Ferrari, A. C.; Robertson, J. Phys. Rev. Lett. 2004, 93, 185503.

(23) Lazzeri, M.; Piscanec, S.; Mauri, F.; Ferrari, A. C.; Robertson, J. Phys. Rev. B 2006, 73, 155426.

(24) Das, A.; Sood, A. K.; Govindaraj, A.; Saitta, A. M.; Lazzeri, M.; Mauri, F.; Rao, C. N. R. Phys. Rev. Lett. 2007, 99, 136803.

(25) Tsang, J. C.; Freitag, M.; Perebeinos, V.; Liu, J.; Avouris, P. Nat. Nanotechnol. 2007, 2, 725-

730.

(26) Kalbac, M.; Farhat, H.; Kavan, L.; Kong, J.; Dresselhaus, M. S. Nano Lett. 2008, 8, 3532-3537.

(27) www.nanocarblab.com.

(28) www.cheaptubes.com.

(29) Takenobu, T.; Takano, T.; Shiraishi, M.; Murakami, Y.; Ata, M.; Kataura, H.; Achiba, Y.; Iwasa, Y. Nat. Mater. 2003, 2, 683-688.

(30) Jeanguillaume, C.; Colliex, C. Ultramicroscopy 1989, 28, 252-257.

(31) Arenal, R.; de la Pena, F.; Stephan, O.; Walls, M.; Tence, M.; Loiseau, A.; Colliex, C. Ultramicroscopy 2008, 109, 32-38.

(32) Lin, H.; Arenal, R.; Enouz-Vedrenne, S.; Stephan, O.; Loiseau, A. J. Phys. Chem. C 2009, 113, 9509-9511.

(33) Arenal, R.; Stephan, O.; Bruno, P.; Gruen, D. M. Appl. Phys. Lett. 2009, 94, 111905.

(34) Ayala, P.; Arenal, R.; Rummeli, M.; Rubio, A.; Pichler, T. Carbon 2010, 48, 575-586. 
(35) Bachilo, S. M.; Strano, M. S.; Kittrell, C.; Hauge, R. H.; Smalley, R. E.; Weisman, R. B. Science 2002, 298, 2361-2366.

(36) Li, L. J.; Khlobystov, A. N.; Wiltshire, J. G.; Briggs, G. A. D.; Nicholas, R. J. Nat. Mater. 2005, 4, 481-485.

(37) Yanagi, K.; Iakoubovskii, K.; Matsui, H.; Matsuzaki, H.; Okamoto, H.; Miyata, Y.; Maniwa, Y.; Kazaoui, S.; Minami, N.; Kataura, H. J. Am. Chem. Soc. 2007, 129, 4992-4997.

(38) Bantignies, J. L.; Sauvajol, J. L.; Rahmani, A.; Flahaut, E. Phys. Rev. B 2006, 74, 195424.

(39) Sbai, K.; Rahmani, A.; Chadli, H.; Bantignies, J. L.; Hermet, P.; Sauvajol, J. L. J. Phys. Chem. B 2006, 110, 12388-12393.

(40) Hernandez, V.; Casado, J.; Ramirez, F. J.; Zotti, G.; Hotta, S.; Navarrete, J. T. L. J. Chem. Phys. 1996, 104, 9271-9282.

(41) Casado, J.; Hernandez, V.; Hotta, S.; Navarrete, J. T. L. J. Chem. Phys. 1998, 109, 1041910429.

(42) Casado, J.; Bengoechea, M.; Navarrete, J. T. L.; Otero, T. F. Synth. Met. 1998, 95, 93-100. 